\title{
25 Research Soure \\ CXCR4-CXCL12 axis and distant metastatic outgrowth in head and neck malignancy
}

\section{Yin Li}

Klinikum rechts der Isar der Technischen Universitat Munchen Klinik und Poliklinik fur Hals- Nasen- und Ohrenheilkunde

\section{Leila Bahadori}

Klinikum rechts der Isar der Technischen Universitat Munchen Klinik und Poliklinik fur Hals- Nasen- und Ohrenheilkunde

\section{Kristin Fritsche}

Klinikum rechts der Isar der Technischen Universitat Munchen Klinik und Poliklinik fur Hals- Nasen- und Ohrenheilkunde

\section{Percy Knolle}

Klinikum rechts der Isar der Technischen Universitat Munchen

\section{Achim Krüger}

Klinikum rechts der Isar der Technischen Universitat Munchen

\section{Henning Bier}

Klinikum rechts der Isar der Technischen Universitat Munchen

\section{Andreas Knopf ( $\square$ knopf.ent@gmail.com )}

Albert-Ludwigs-Universitat Freiburg https://orcid.org/0000-0001-5196-1803

\section{Research article}

Keywords: XCR4, CXCL12, head and neck manlignancy, recurrency, metastasis

Posted Date: May 1st, 2020

DOI: https://doi.org/10.21203/rs.3.rs-24735/v1

License: (9) (i) This work is licensed under a Creative Commons Attribution 4.0 International License. Read Full License 


\section{Abstract}

Background: To analyze whether distant metastatic outgrowth in different head and neck malignancies (HNM) underlies the CXCR4-CXCL12 axis as overriding molecular mechanism.

Methods: Clinic-pathological data of 1,250 HNM was included. HNM were collected due to different capability to exhibit distant metastasis comprising basal cell, squamous cell, and adenoid-cystic carcinoma as well as melanoma. MMP2/9, TIMP1/2, CXCR4, and CXCL12 immunohistochemistry was done in 190 randomly selected specimens.

Results: Immunohistochemistry visualized a significant increase in MMP2/9, TIMP1/2, CXCR4, and CXCL12 protein expression following the clinical occurrence of distant metastasis. CXCR4, CXCL12, and TIMP2-expression significantly increases with number of affected organs by distant metastasis. Cox regression demonstrated CXCR4-overexpression and advanced T-status being independent risk factors of distant metastasis associated death.

Conclusion: The CXCR4-CXCL12 axis is associated with the occurrence of distant metastases in different HNM. The increased risk of distant metastasis associated death was identified at primary tumour site and, therefore, potentially influences further treatment protocols.

\section{Introduction}

Head and neck malignomas (HNM) consist of a broad variety of malignant tumours with different histological subtypes and distinct clinical behavior. Risk factors range from ultraviolet irradiation in basal cell carcinoma (BCC) and malignant melanoma (MM), nicotine and alcohol abuse as well as HPVinfection in head and neck squamous cell carcinoma (HNSCC) to unknown predisposing factors in adenoid-cystic carcinomas (ACC) [1-4]. Subsequently, molecular mechanisms underlying the respective entity vary. However, in all HNM patient's prognosis is inherently associated with the TNM status. Thereby, the occurrence of loco-regional lymph node and particularly distant metastasis is associated with a significantly reduced survival $[5,6]$. The current study analyzes whether different HNM share overriding molecular mechanisms that are associated with distant metastatic outgrowth. Therefore, different HNM were selected due to their clinical behavior to develop distant metastasis. BCC is a slowly progressive, locally destructive skin cancer that accounts for almost $80 \%$ of all non-melanoma skin cancers. The incidence of metastases in BCC is estimated to be about $0.0028-0.55$ of all BCC cases [7]. HNSCC is the sixth common malignant neoplasm worldwide, accounting for more than $90 \%$ of head and neck cancers [8]. Overall, the loco-regional lymph node metastasis rate is estimated to be about $60 \%$ [9]. Distant metastases were diagnosed in 10-20\% [9-12]. MM originates from melanocytes and accounts for $4 \%$ of skin malignancies. MM represents the most aggressive skin cancer and usually accompanies the initiation of loco-regional and distant metastasis with a dramatically reduced 5-year survival of $<12 \%[13$, 14]. ACC is a frequent malignant tumour of salivary glands, which is characterized by frequent local recurrences and almost exclusive distant metastasis [15]. ACC usually develops late-onset distant 
metastases, years after resection of the primary tumour [16]. The current study focuses on the CXCR4CXCL12 axis as overriding molecular mechanism in distant metastatic outgrowth that was suggested to be associated with poor survival in BCC, HNSCC, MM, and ACC [17-22].

\section{Material And Methods}

\section{Patient selection}

The current study includes a total of 1,250 HNM patients that were diagnosed in the ENT department of the University Hospital Rechts der Isar, Munich. Thirty-one patients with BCC, 1,057 patients with HNSCC, 146 patients with MM, and 16 patients with ACC were included. Tumour samples were histologically reviewed by at least two experienced pathologists. Dysplasia, carcinoma in situ, and other histologic subtypes were excluded. Clinical parameters and survival data were retrospectively collected: age, sex, TNM-Staging, grading, treatment modalities, recurrence, and death/loss to follow-up. Patients with lacking data, incomplete staging, and refused/not finished surgical and/or conservative treatment (radio-/chemotherapy) were excluded from survival analysis. The study was approved by the ethical committee, Technical University Munich (08/14/2019, 418/19S).

\section{Immunohistochemistry}

Tumour samples were achieved from primary tumour sites at the time of diagnosis. Paraffin-embedded tumour (FFPE) samples from $31 \mathrm{BCC}, 118 \mathrm{HNSCC}, 32 \mathrm{MM}$, and nine ACC were randomly selected and analyzed via immunohistochemistry (IHC). FFPE tumor sections $(2.5 \mu \mathrm{m})$ were MMP2 (DCS Innovative Diagnostik-Systeme, Hamburg, Germany, 1:100), MMP9 (Biomol GmbH, Hamburg, Germany, 1:1000), TIMP1 (R\&D, Wiesbaden, Germany, 1:500), TIMP2 (Biomol, 1:500), CXCR4 (R\&D, 1:200), and CXCL12 (R\&D, 1:1000) immuno-stained and visualized with the Bond Polymer Refine Detection Kit (Leica, Nussloch, Germany). Expression levels were classified using a scoring system analyzing the staining intensity ( $0=$ no staining, $1=$ low, $2=$ moderate, $3=$ strong staining intensity) and the relative proportion of stained cells $(0,1=<10 \%, 2=10-39 \%, 3=40-69 \%, 4=>70$ of the tumor cells). A cumulative score (range $0-7$ points) was assessed by adding both scores. A positive staining was defined by a cumulative score equal or greater than 3 .

\section{Statistical analysis}

Differences between the groups were analyzed using the Chi square test and Fisher exact test for categorical, and the unpaired student's t-test for continuous variables. For the analysis of the independent groups, the one-way ANOVA tests were used. As main endpoint, the overall survival (OS) was assessed measuring the time from treatment to death of any cause. Survival rates and curves were calculated and illustrated by the Kaplan-Meier method and further analyzed by the log-rank test. Variables that revealed prognostic or effect modifying potential on the outcome were subsequently evaluated by the proportional Cox regression for forward selection. p-values $<0.05$ were considered statistically significant. Statistical analysis was done using SPSS (SPSS Inc., Chicago, IL). 


\section{Results}

\section{Demographic and tumour characteristics of analyzed HNC}

The overall cohort demonstrated significant differences in the age and gender distribution. While HNSCC, $\mathrm{MM}$, and ACC demonstrated a mean age of 60 years, BCC patients were significantly older (72 years; $p<0.0001)$. Our study demonstrated a significant predominance of male patients in BCC (2.5:1) and HNSCC (4:1). MM showed a more balanced gender distribution (1.3:1), while a female predominance was observed in ACC $(1.6: 1 ; p<0.0001)$. The current study, performed in a tertiary ear nose throat department, revealed that the majority (88\%) of ACC originated in major salivary glands (parotid and submandibular gland), BCC originated in head and neck cutis (100\%). The majority of head and neck MM were diagnosed in the cutis (88\%), while $10 \%$ originated in the sinonasal system, one percent in the oral cavity, and one percent in the nasopharynx, respectively. HNSCC demonstrated a pronounced occurrence in the oropharynx (39\%), followed by the hypopharynx (21\%), larynx (20\%), and the oral cavity (15\%) (Table 1$)$.

In BCC, the vast majority of patients presented limited T-status (T1/2: 97\%) and underwent primary tumour resection. R0 status was achieved in $97 \%$ of our patients. None of our BCC patients suffered from lymph node and/or distant metastases at the time of diagnosis or during the follow up period. HNSCC exhibited a balanced T-status (T1/2: $56 \%$; T3/4: 44\%). Head and neck surgery in HNSCC (66\%) was sustained by adjuvant radio-(chemo-) therapy in $47 \%$ of our patients. R0-status was achieved in $83 \%$ of our HNSCC patients, while $10 \%$ remained insufficiently resected. In MM, $74 \%$ of our patients showed limited T-Status (T1/2). Twenty-six percent of our patients suffered from T3/4 MM that usually refers to a mucosal subtype with a missing T1/2-status in the UICC classification system. $64 \%$ of our patients underwent solitary tumour resection, while combined treatment regimens were applied in $36 \%$. Tumour debulking was done in $8 \%$ of the MM cohort, resulting in a R2 status. Tumour surgery with adjuvant radiotherapy was done in $81 \%$ of the ACC patients. Despite radical parotid and submandibular gland surgery, tumour margins remained microscopically non-in-sano in $21 \%$ of our patients (Table 1 ).

\section{Distant metastatic profile}

The distant metastatic profile differed significantly between the analyzed groups. At the time of diagnosis, distant metastases were diagnosed in $4 \%$ of HNSCC, $6 \%$ of ACC, and $10 \%$ of MM ( $p=0.01)$. Metachronous metastases occurred in 14 of HNSCC, $19 \%$ of ACC, and $24 \%$ of MM resulting in an overall metastasis rate of $18 \%$ in HNSCC, $25 \%$ of $A C C$, and $34 \%$ of $\mathrm{MM}(\mathrm{p}=0.0009)$. Loco-regional and distant metastasis did not occur in BCC. Time from first diagnosis to the occurrence of metachronous metastases ranged from 13 to 37 months, without differences between the groups $(p=0.6)$. The mean follow up time was $\geq 60$ months for all analyzed tumour entities. There were significant differences in the number and distribution of affected organs. While metastases in more than one organ occurred in $18 \%$ of the HNSCC and $25 \%$ of ACC, MM demonstrated multi-organ manifestation in $43 \%$ of our cases $(\mathrm{p}<0.0001)$. The lungs represented the most affected organs in ACC $(75 \%)$ and HNSCC (48\%). The liver and bone were involved in $18 \%$ and $17 \%$ of all HNSCC patients, while the distant metastatic spread in 
other organs occurred infrequently. In contrast, there was a balanced and extensive affection of different distant metastasis organs in MM ( $p=0.04$; Table 2).

Impact of the CXCR4-CXCL12-axis in HNC distant metastases

There were significant differences in the expression patterns of analyzed proteins that are associated with the CXCR4-CXCL12 axis. Immunohistochemical (IHC) analysis visualized a stepwise increase of MMP2 and 9 expression from BCC, HNSCC, and MM to ACC ( $p<0.0001)$. TIMP1 was visualized in all tumour entities with an increased expression in MM and ACC $(p<0.0001)$. There were striking differences in the expression of TIMP2, CXCR4, and CXCL12 with significant higher expression levels in MM and ACC (all: $p<0.0001$; Figure 1).

IHC scoring visualized significant differences in the TIMP2, CXCR4, and CXCL12 expression with respect to the number of organs affected by distant metastasis $(p=0.02 ; p=0.002 ; p=0.047$; Figure 2$)$. Even MMP9 and TIMP1 showed a stepwise increase in protein expression with the lowest level in M0 and the highest level when three or more organ systems were affected. However, the trend failed to achieve statistical significance (Figure 2).

Analysis of the overall survival demonstrated a significantly better outcome of M0 individuals (mean: 254 months) when compared with their M1 counterparts (mean: 44 months) ( $p<0.0001$; Figure 3 ). Forward selected, M0/1 adjusted, proportional Cox regression of survival modifying parameters (T, N, MMP2/9, TIMP1/2, CXCR4) identified CXCR4 positivity at primary tumor site (HR: 3.57; $p=0.017$ ) and increasing T status (HR: $1.823 ; p=0.025)$ being solitary survival modifying parameters in HNM.

CXCR4 expression levels at primary tumour site differed significantly due to the location of distant metastasis. Highest CXCR4 levels were detected in patients with skin and CNS metastases, moderate levels in patients with lung and liver involvement, and low CXCR4 levels in all other locations $(p=0.04$; Figure 4).

\section{Discussion}

Head and neck malignancies (HNM) represent a melting pot of different malignant lesions with distinct clinical behavior. Risk factors and molecular mechanisms underlying the respective entity differ significantly. However, malignant capacity is clinically characterized by an infiltrative and destroying growing pattern at primary tumour site, locoregional lymph node manifestation, and the exhibition of distant metastases. Particularly, the occurrence of distant metastases is inherently associated with reduced patients' survival and usually results in palliative treatment regimens. At the time of primary diagnosis, the reliable identification of patients with increased risk to develop distant metastasis is major clinical impact in order to modify treatment recommendations. The current study analyzes the CXCR4CXCL12-axis as overriding molecular mechanism in the distant metastatic outgrowth of different HNM. HNM were selected due to a distinct clinical metastatic profile. Concordant with the present literature, our study did not observe loco-regional or distant metastases in BCC [7]. While loco-regional metastases were 
diagnosed in $60 \%$ of the HNSCC and MM at the time of diagnosis, lymph node metastases were not seen in ACC. The occurrence of distant metastases differed significantly between the groups. At the time of diagnosis, distant metastases were diagnosed in $4 \%$ of HNSCC, $6 \%$ of ACC, and $10 \%$ of MM ( $p=0.01$ ). Metachronous metastases occurred in 14 of HNSCC, $19 \%$ of ACC, and $24 \%$ of MM resulting in an overall metastasis rate of $18 \%$ in HNSCC, $25 \%$ of ACC, and $34 \%$ of MM ( $p=0.0009)$. The aggressive clinical phenotype in MM was highlighted by a high proportion of multiple organ manifestation. While HNSCC and ACC demonstrated multiple organ manifestation in $18 \%$ and $25 \%$ of the analyzed cases, multilocularity was observed in $43 \%$ of our MM patients. The lungs were predominantly affected in HNSCC and ACC. MM showed an extensive metastatic spread with systemic disease manifestation in many organs. Several studies demonstrated the association of the CXCR4-CXCL12 axis and clinicopathological features of malignancy [23-25]. In our study, the protein expression of CXCR4, CXCL12, TIMP1, and TIMP2 was significantly higher in MM and ACC when compared with HNSCC. BCC, that did not develop loco-regional or distant metastasis, showed the lowest expressions of the analyzed proteins. Recently, MMPs, TIMPs, CXCR4, and CXCL12 were associated with distant metastatic capacity [26-29]. Moreover囚a CXCL12 dependent increase of MMP2 and MMP9 secretion by activating ERK-1/2 signaling pathway was demonstrated [30, 31]. Interestingly, our study demonstrated that patients with distant metastases in three or more organs showed significantly higher TIMP1, CXCR4, and CXCL12 protein expression in tumor samples at initial diagnosis than patients with distant metastases in 1-2 organs, or particularly than patients without distant metastases. Although the lung was the most affected metastatic organ in HNSCC and ACC, the CXCR4 expression in primary tumors did not show the organspecificity.

A pooled model from meta-analysis showed a significant shorter OS in HNM patients with CXCR4 overexpression ( $\mathrm{HR}=2.02,95 \% \mathrm{Cl}, 1.37-2.97$ ) [32]. Accordingly, forward selected, M0/1 adjusted, proportional Cox regression of survival modifying parameters ( $T, N, R, M M P 2 / 9, T I M P 1 / 2$ and CXCR4) identified CXCR4 positivity being a solitary survival modifying parameter in HNM. In a large study of $233 \mathrm{HNSCC}$ patients with inoperable tumors undergoing primary RT/RT-CT CXCR4 expression was also associated with increased risk of distant metastasis [33]. Recently囚a multicenter study revealed that intracellular CXCL12 expression was associated with lower loco-regional control after postoperative RT-CT in a cohort of $221 \mathrm{HNSCC}$ patients [34]. Consistent with our findings, McConnell et al. demonstrated that patients with AJCC stage II melanomas had 3-fold risk of disease reoccurrence in individuals with high total CXCR4 expression (>50\%). Nevertheless, CXCL12 over-expression in the adjacent epidermis of all melanoma stages with tumour progression was associated with increased time to metastasis [35]. Due to the autocrine and paracrine CXCL12 secretion, the contradictory phenomenon remains to be further studied. In primary nasal-surface BCC tumor, upregulation of CXCR4 in normal skin tissues compared to normal skin tissues revealed that potential capacity of CXCR4 in progression and invasion of nasalsurface BCC [22]. Bazal et al. demonstrated that BCC patients with deep tissue invasion showed significant high CXCR4 staining rate (75\%) than patients with papillary dermis invasion (14.3\%) [36]. In our study Cox regression showed that CXCR4 expression and T1/2 vs. T3/4 can be solitary predictors for overall survival. 


\section{Conclusion}

The CXCR4-CXCL12 axis plays a pivotal role in the distant metastatic capacity of different HNM. The increased risk of distant metastasis associated death can be identified at primary tumour site and, therefore, potentially influences further treatment protocols. Functional studies have to investigate the interaction of CXCL12 and CXCR4 in HNM.

a

\section{Declarations}

Ethics approval and consent to participate: This article does not contain any studies with human participants or animals performed by any of the authors. The study was approved by the local ethical committee.

Consent for publication: All authors gave consent for publication.

Competing interests: Authors disclose any financial and personal relationships with other people or organisations that could inappropriately influence their work.

Funding: Authors did not receive any funding associated with the current manuscript.

Authors' contributions: Conception: YL, LB, KF, PK, AKr, HB, AK; experiments: YL, LB KF, AK; data acquisition: YL, LB, KF, PK, AKr, HB, AK; interpretation: YL, LB, KF, PK, AKr, HB, AK; analysis: YL, LB KF, AK; manuscript preparation; $Y L, K F, A K$; manuscript correction: $Y L, L B, K F, P K, A K r, H B, A K$

Acknowledgements: -/-

Supporting data / deposition: There is no supporting data attached.

\section{References}

[1] Lewis A, Kang R, Levine A, Maghami E. The New Face of Head and Neck Cancer: The HPV Epidemic. Oncology (Williston Park). 2015;29:616-26.

[2] Chinem VP, Miot HA. Epidemiology of basal cell carcinoma. An Bras Dermatol. 2011;86:292-305.

[3] Ankeny JS, Labadie B, Luke J, Hsueh E, Messina J, Zager JS. Review of diagnostic, prognostic, and predictive biomarkers in melanoma. Clin Exp Metastasis. 2018.

[4] Syrjanen S, Rautava J, Syrjanen K. HPV in Head and Neck Cancer-30 Years of History. Recent Results Cancer Res. 2017;206:3-25.

[5] Johnson JT, Barnes EL, Myers EN, Schramm VL, Jr., Borochovitz D, Sigler BA. The extracapsular spread of tumors in cervical node metastasis. Arch Otolaryngol. 1981;107:725-9. 
[6] Richard JM, Sancho-Garnier H, Micheau C, Saravane D, Cachin Y. Prognostic factors in cervical lymph node metastasis in upper respiratory and digestive tract carcinomas: study of 1,713 cases during a 15year period. Laryngoscope. 1987;97:97-101.

[7] Seo SH, Shim WH, Shin DH, Kim YS, Sung HW. Pulmonary metastasis of Basal cell carcinoma. Ann Dermatol. 2011;23:213-6.

[8] Jemal A, Siegel R, Ward E, Hao Y, Xu J, Thun MJ. Cancer statistics, 2009. CA Cancer J Clin. 2009;59:225-49.

[9] Leemans CR, Tiwari R, Nauta JJ, van der Waal I, Snow GB. Regional lymph node involvement and its significance in the development of distant metastases in head and neck carcinoma. Cancer. 1993;71:4526.

[10] Gourin CG, Johnson JT. Surgical treatment of squamous cell carcinoma of the base of tongue. Head Neck. 2001;23:653-60.

[11] Harrison LB, Lee HJ, Pfister DG, Kraus DH, White C, Raben A, et al. Long term results of primary radiotherapy with/without neck dissection for squamous cell cancer of the base of tongue. Head Neck. 1998;20:668-73.

[12] Al-Othman MO, Morris CG, Hinerman RW, Amdur RJ, Mendenhall WM. Distant metastases after definitive radiotherapy for squamous cell carcinoma of the head and neck. Head Neck. 2003;25:629-33.

[13] de Snoo FA, Hayward NK. Cutaneous melanoma susceptibility and progression genes. Cancer Lett. 2005;230:153-86.

[14] Thompson JA. Ten years of progress in melanoma. J Natl Compr Canc Netw. 2012;10:932-5.

[15] Knopf A, Szyper M, Mansour N, Sonnenberg J, Hofauer B, Niedermeyer H. A critical review of 20 years of parotid gland surgery. Acta Otolaryngol. 2016;136:711-6.

[16] Bonaparte JP, Hart R, Trites J, Taylor MS. Incidence of adenoid cystic carcinoma in nova scotia: 30year population-based epidemiologic study. J Otolaryngol Head Neck Surg. 2008;37:642-8.

[17] Albert S, Riveiro ME, Halimi C, Hourseau M, Couvelard A, Serova M, et al. Focus on the role of the CXCL12/CXCR4 chemokine axis in head and neck squamous cell carcinoma. Head Neck. 2013;35:181928.

[18] Zushi Y, Noguchi K, Hashitani S, Sakurai K, Segawa E, Takaoka K, et al. Relations among expression of CXCR4, histological patterns, and metastatic potential in adenoid cystic carcinoma of the head and neck. Int J Oncol. 2008;33:1133-9. 
[19] Kuhnelt-Leddihn L, Muller H, Eisendle K, Zelger B, Weinlich G. Overexpression of the chemokine receptors CXCR4, CCR7, CCR9, and CCR10 in human primary cutaneous melanoma: a potential prognostic value for CCR7 and CCR10? Arch Dermatol Res. 2012;304:185-93.

[20] Knopf A, Bahadori L, Fritsche K, Piontek G, Becker CC, Knolle P, et al. Primary tumor-associated expression of CXCR4 predicts formation of local and systemic recurrency in head and neck squamous cell carcinoma. Oncotarget. 2017;8:112739-47.

[21] Knopf A, Fritsche MK, Li Y. CXCR4: A New Player in an Old Scene? ORL J Otorhinolaryngol Relat Spec. 2017;79:34-42.

[22] Xu CZ, Wang PH, Yan XJ, Wang T, Chen D, Zhang ZJ, et al. Expression of CXCR4 is associated with progression and invasion in patients with nasal-surface basal cell carcinoma. ORL J Otorhinolaryngol Relat Spec. 2013;75:332-41.

[23] Meng X, Wuyi L, Yuhong X, Xinming C. Expression of CXCR4 in oral squamous cell carcinoma: correlations with clinicopathology and pivotal role of proliferation. J Oral Pathol Med. 2010;39:63-8.

[24] Wald O, Pappo O, Safadi R, Dagan-Berger M, Beider K, Wald H, et al. Involvement of the CXCL12/CXCR4 pathway in the advanced liver disease that is associated with hepatitis $C$ virus or hepatitis B virus. Eur J Immunol. 2004;34:1164-74.

[25] Liu H, Pan Z, Li A, Fu S, Lei Y, Sun H, et al. Roles of chemokine receptor 4 (CXCR4) and chemokine ligand 12 (CXCL12) in metastasis of hepatocellular carcinoma cells. Cell Mol Immunol. 2008;5:373-8.

[26] Mendes O, Kim HT, Stoica G. Expression of MMP2, MMP9 and MMP3 in breast cancer brain metastasis in a rat model. Clin Exp Metastasis. 2005;22:237-46.

[27] Uchida D, Onoue T, Tomizuka Y, Begum NM, Miwa Y, Yoshida H, et al. Involvement of an autocrine stromal cell derived factor-1/CXCR4 system on the distant metastasis of human oral squamous cell carcinoma. Mol Cancer Res. 2007;5:685-94.

[28] Lim JB, Chung HW. Serum ENA78/CXCL5, SDF-1/CXCL12, and their combinations as potential biomarkers for prediction of the presence and distant metastasis of primary gastric cancer. Cytokine. 2015;73:16-22.

[29] Fuksiewicz M, Kotowicz B, Rutkowski A, Kowalska M. The matrix metalloproteinase-7 and proenzyme of metalloproteinase-1 as a potential marker for patients with rectal cancer without distant metastasis. Tumour Biol. 2015;36:3629-35.

[30] Janowska-Wieczorek A, Matsuzaki A, L AM. The Hematopoietic Microenvironment: Matrix Metalloproteinases in the Hematopoietic Microenvironment. Hematology. 2000;4:515-27. 
[31] Samara GJ, Lawrence DM, Chiarelli CJ, Valentino MD, Lyubsky S, Zucker S, et al. CXCR4-mediated adhesion and MMP-9 secretion in head and neck squamous cell carcinoma. Cancer Lett. 2004;214:23141.

[32] Zhao H, Guo L, Zhao H, Zhao J, Weng H, Zhao B. CXCR4 over-expression and survival in cancer: a system review and meta-analysis. Oncotarget. 2015;6:5022-40.

[33] Rave-Frank M, Tehrany N, Kitz J, Leu M, Weber HE, Burfeind P, et al. Prognostic value of CXCL12 and CXCR4 in inoperable head and neck squamous cell carcinoma. Strahlenther Onkol. 2016;192:47-54.

[34] De-Colle C, Monnich D, Welz S, Boeke S, Sipos B, Fend F, et al. SDF-1/CXCR4 expression in head and neck cancer and outcome after postoperative radiochemotherapy. Clin Transl Radiat Oncol. 2017;5:28-36.

[35] McConnell AT, Ellis R, Pathy B, Plummer R, Lovat PE, O'Boyle G. The prognostic significance and impact of the CXCR4/CXCR7/CXCL12 axis in primary cutaneous melanoma. Br J Dermatol. 2016.

[36] Sivrikoz ON, Uyar B, Dag F, Tasli F, Sanal SM. CXCR-4 and COX-2 expression in basal cell carcinomas and well-differentiated squamous cell carcinomas of the skin; their relationship with tumor invasiveness and histological subtype. Turk Patoloji Derg. 2015;31:30-5.

\section{Tables}

Table 1: Clinical characteristics 


\begin{tabular}{|c|c|c|c|c|c|}
\hline & $\mathrm{BCC}$ & HNSCC & MM & ACC & p-value \\
\hline n & 31 & 1057 & 146 & 16 & \\
\hline Age (years) & & & & & $<0.0001$ \\
\hline Median [25\%; 75\%] & $74[64 ; 81]$ & $60[53 ; 67]$ & $62[49 ; 70]$ & $62[50 ; 71]$ & \\
\hline Mean \pm SD & $72 \pm 13$ & $60 \pm 11$ & $60 \pm 16$ & $60 \pm 12$ & \\
\hline Sex, n (\%) & & & & & $<0.0001$ \\
\hline Male & $22(71)$ & $841(80)$ & $83(57)$ & $6(38)$ & \\
\hline Female & $9(29)$ & $216(20)$ & $63(43)$ & $10(62)$ & \\
\hline \multicolumn{6}{|l|}{ Location } \\
\hline Sinunasal & & $35(3)$ & $15(10)$ & 0 & \\
\hline Nasopharynx & & $20(2)$ & 1 & 0 & \\
\hline Oropharynx & & 415 (39) & 0 & 0 & \\
\hline Hypopharynx & & $220(21)$ & 0 & 0 & \\
\hline Larynx & & $208(20)$ & 0 & $2(12)$ & \\
\hline Oral cavity & & $154(15)$ & 1 & 0 & \\
\hline Head and neck skin & $31(100)$ & 0 & $129(88)$ & 0 & \\
\hline Major salivary glands & & 0 & 0 & $14(88)$ & \\
\hline CUP & & 5 & 0 & 0 & \\
\hline T stage, n (\%) & & & & & $<0.0001$ \\
\hline Tx & 0 & 8 & 0 & 0 & \\
\hline $\mathrm{T} 1$ & $22(71)$ & $276(26)$ & $80(55)$ & $2(11)$ & \\
\hline $\mathrm{T} 2$ & $8(26)$ & $313(30)$ & $17(12)$ & $10(63)$ & \\
\hline T3 & $1(3)$ & $221(21)$ & $29(20)$ & $3(20)$ & \\
\hline $\mathrm{T} 4$ & 0 & $239(23)$ & $20(14)$ & $1(6)$ & \\
\hline N stage, n (\%) & & & & & $<0.0001$ \\
\hline NO & $31(100)$ & $417(40)$ & $59(40)$ & $16(100)$ & \\
\hline $\mathrm{N}+$ & 0 & $640(60)$ & $87(60)$ & 0 & \\
\hline M stage, n (\%) & & & & & 0.01 \\
\hline MO & $31(100)$ & $1015(96)$ & $132(90)$ & $15(94)$ & \\
\hline
\end{tabular}


$\begin{array}{llll}\text { M1 } & 0 & 42(4) & 14(10)\end{array}$

\section{Grading, n (\%)}

$\begin{array}{ll}\text { G1 } & 45(4) \\ \text { G2 } & 506(48) \\ \text { G3 } & 469(45) \\ \text { G4 } & 13(1) \\ \text { Gx } & 24(2)\end{array}$

\section{R stage, $n$ (\%)}

0.28

\begin{tabular}{lllll} 
R0 & $30(97)$ & $590(83)$ & $11(92)$ & $10(71)$ \\
R1 & $1(3)$ & $58(8)$ & 0 & $3(21)$ \\
\hline R2 & 0 & $14(2)$ & $1(8)$ & 0 \\
\hline Rx & 0 & $50(7)$ & & $1(7)$
\end{tabular}

Treatment, $\mathbf{n}(\%)$

$<0.0001$

\begin{tabular}{lllll} 
OP only & $30(97)$ & $187(19)$ & $93(64)$ & $1(6)$ \\
\hline OP + RTX & 0 & $300(28)$ & $16(11)$ & $13(81)$ \\
\hline OP + RCTX & 0 & $203(19)$ & $12(8)$ & 0 \\
\hline OP + CTX & 0 & 0 & $15(10)$ & 0 \\
\hline Prim. RCTX & 0 & $329(31)$ & 1 & 0 \\
\hline Prim. RTX & $1(3)$ & $36(3)$ & 1 & $2(13)$ \\
\hline Immunotherapy & 0 & 0 & $9(6)$ & 0
\end{tabular}

Table 2: Distant metastatic profile 
HNSCC MM ACC p-value

\section{M stage, $n(\%)$}

\begin{tabular}{lllll}
\hline M1 synchronous & $42(4)$ & $14(10)$ & $1(6)$ & 0.01 \\
\hline M1 metachronous & $143(14)$ & $35(24)$ & $3(19)$ & 0.0009 \\
\hline
\end{tabular}

Time frame, Mean \pm SD

Diagnosis to metachronous M1

$18 \pm 14 \quad 37 \pm 33 \quad 13 \pm 5 \quad 0.6$

Location, n (\%)

\begin{tabular}{lllll} 
Multi-organ disease & $34(18)$ & $21(43)$ & $1(25)$ & $<0.0001$ \\
\hline Liver & $34(18)$ & $15(31)$ & $1(25)$ & 0.04 \\
\hline Lung & $88(48)$ & $18(37)$ & $3(75)$ & \\
\hline Mediastinum & $12(7)$ & $1(2)$ & 0 & \\
\hline Bone & $32(17)$ & $7(14)$ & 0 & \\
\hline Skin & $16(9)$ & $5(10)$ & 1 & \\
Central nervous system & $7(4)$ & $16(33)$ & 0 & \\
Peripheral lymph nodes & $12(7)$ & $20(41)$ & 0 & \\
Other & $24(13)$ & $1(2)$ & 0 &
\end{tabular}

\section{Figures}

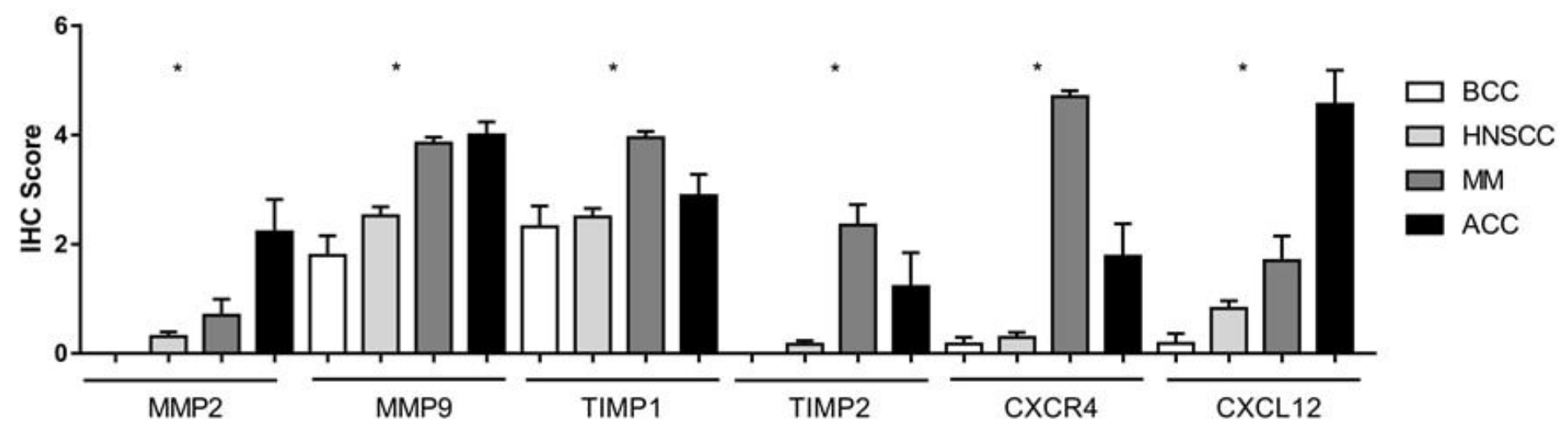

Figure 1

Immunohistochemical markers in HNC primary tumors Immunohistochemistry of primary tumor specimens visualizes a stepwise increase of all analyzed proteins following the clinical occurrence of distant metastasis. 


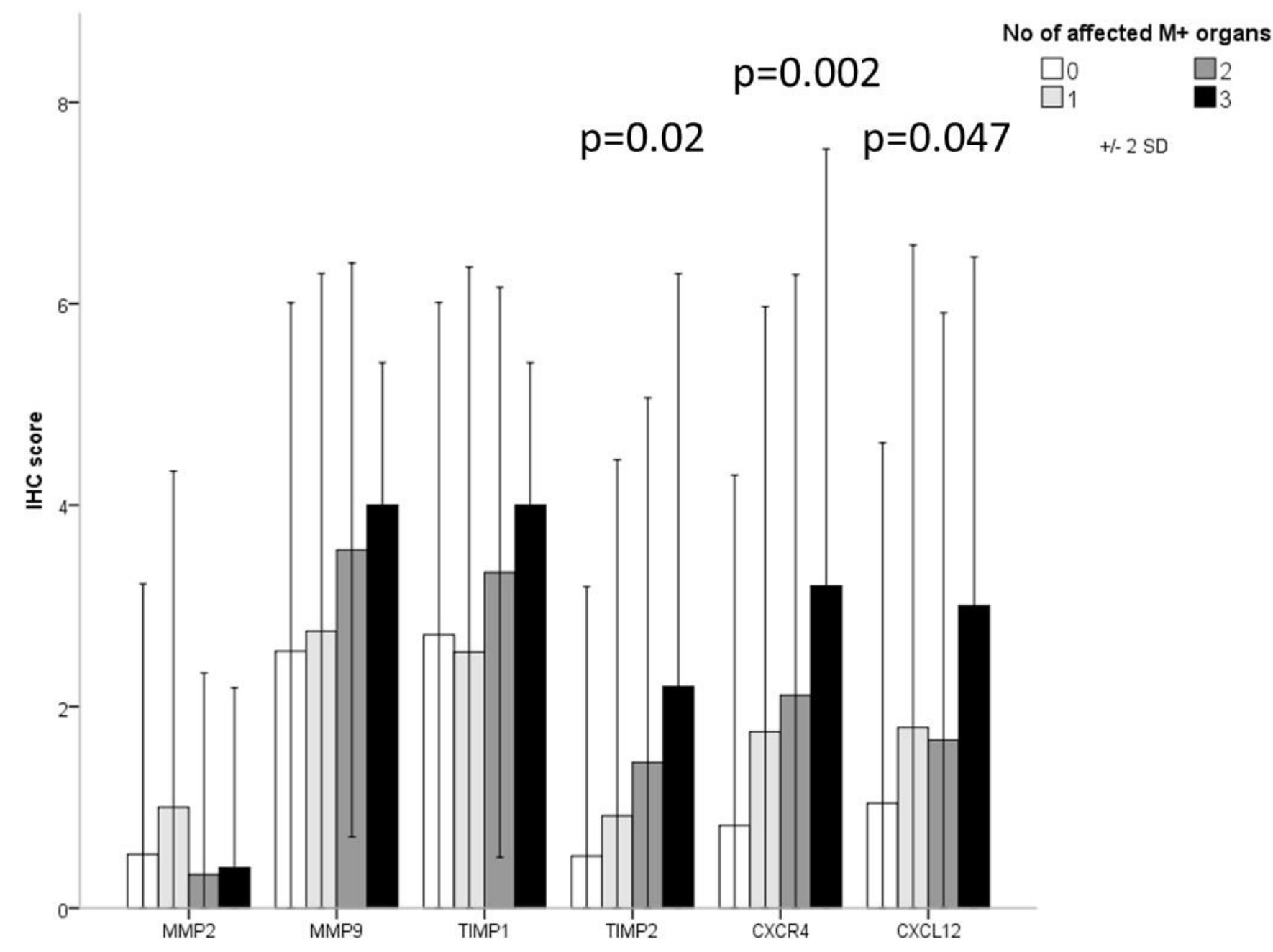

Figure 2

Immunohistochemical markers due to the number of affected organs Immunohistochemistry of primary tumor specimens visualizes a significant increase in TIMP2, CXCR4, and CXCL12 expression with the number of distant metastasis affected organs. 


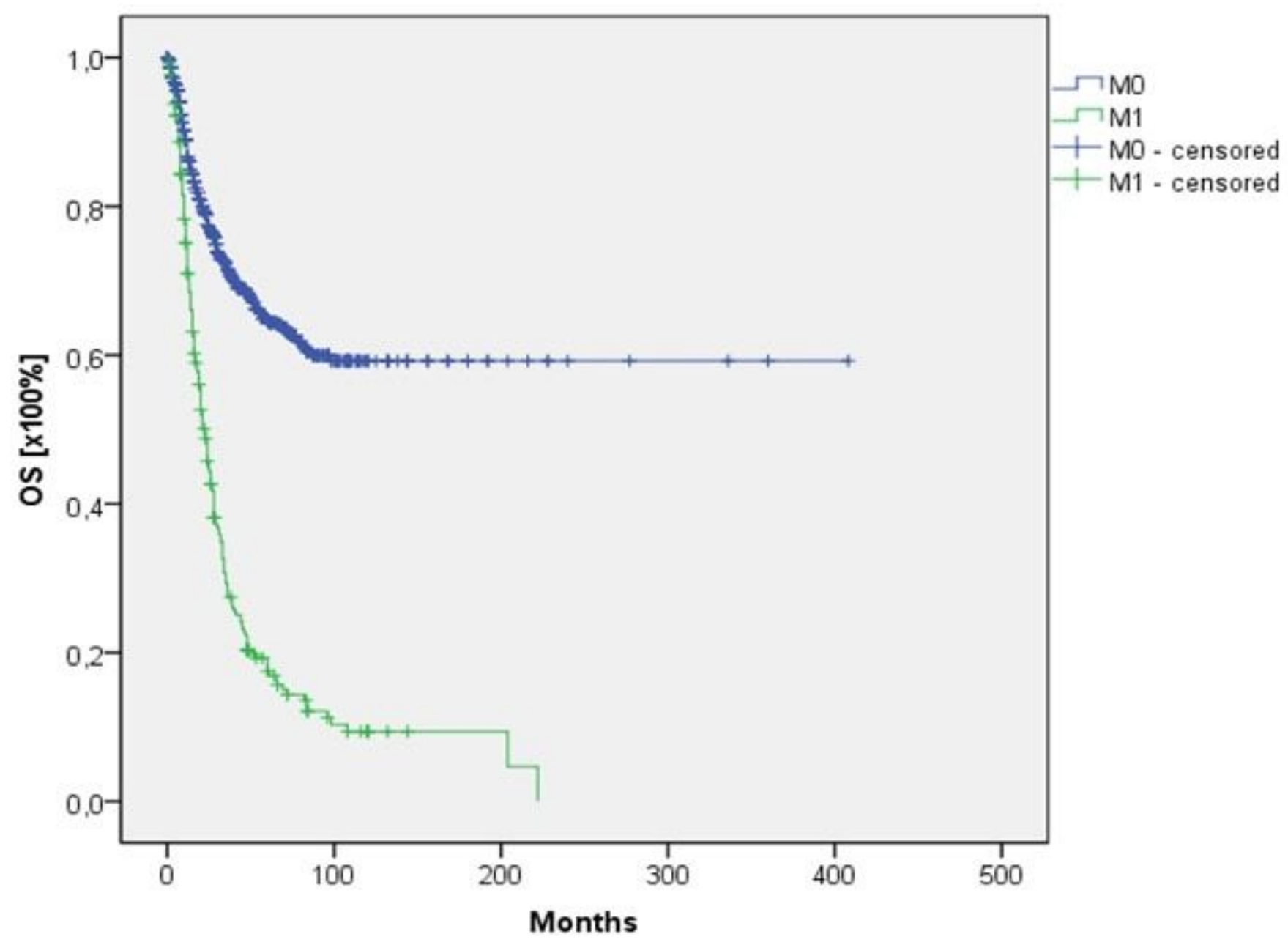

Figure 3

Overall survival in metastasizing HNM Overall survival (OS) was significantly better in patients without distant metastasis when compared with their M1 counterparts.

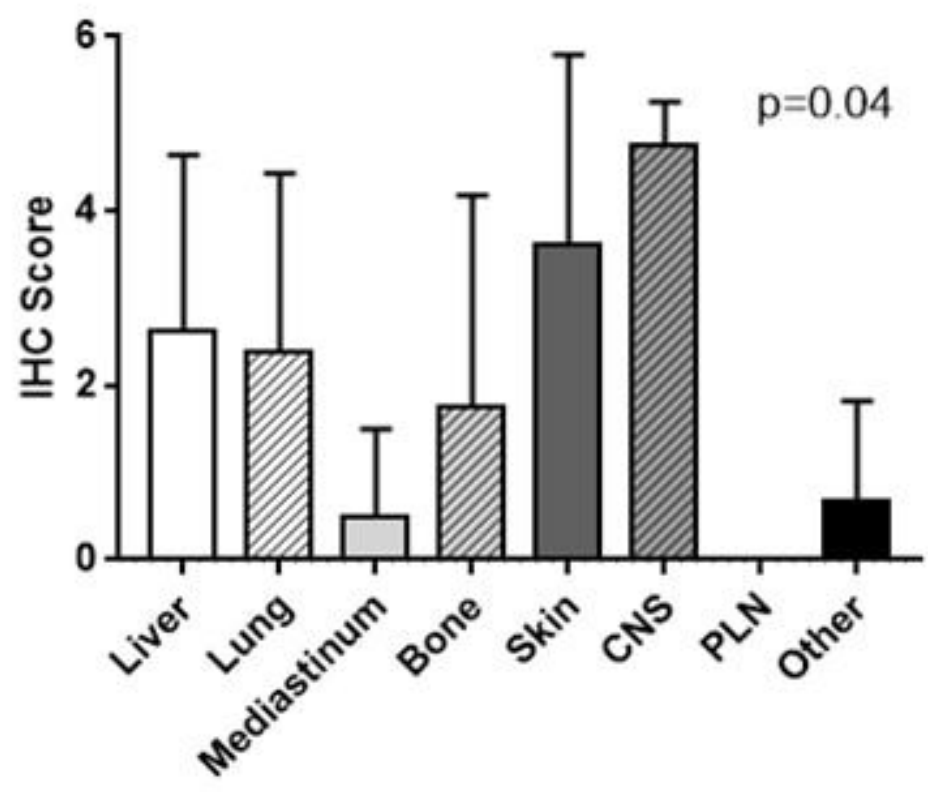




\section{Figure 4}

Immunohistochemical markers in different distant metastasis organs CXCR4 expression at primary tumour site differed significantly due to the distant metastasis location. Highest CXCR4 levels were detected in skin and CNS metastases, moderate levels in lung and liver involvement, and low CXCR4 levels in all other locations. 